\title{
Carpoteca: ferramenta de ensino em botânica
}

\section{RESUMO}

Ykaro Richard Oliveira ykroliveira@gmail.com 000-0002-9382-5583 Universidade Estadual de Feira de Santana, Feira de Santana, Bahia, Brasil.

Paulo Henrique da Silva paulohenriquemh1@gmail.com 0000-0003-2027-4925 Universidade Federal do Piauí, Picos, Piauí, Brasil.

\section{Maria do Socorro Meireles de}

Deus

socorro meireles@yahoo.com.br 0000-0003-2143-6885

niversidade Federal do Piauí, Picos, Piauí, Brasil.

Nilda Masciel Neiva Gonçalves nildamas@uol.com.br

Universidade Federal do Piauí, Picos,

Piauí, Brasil.

Maria Carolina de Abreu

mariacarolinabreu@hotmail.com

0000-0001-8206-7273

Universidade Federal do Piauí, Picos, Piauí, Brasil.

\begin{abstract}
O ensino da botânica é realizado com uma abordagem tradicional provocando desinteresse nos alunos, sendo marcado ainda pela carência ou má utilização de recursos didáticos ou pouca interação entre os alunos e as plantas. Assim, o objetivo desse trabalho foi confeccionar uma carpoteca com o intuito de investigar a eficiência dessa coleção botânica no processo de ensino aprendizagem acerca do estudo dos frutos. Pelo método de caminhada, foram coletados frutos no município de Picos-Piauí e com turmas do ensino médio, 60 alunos foram divididos em quatro grupos e passaram por tratamentos diferenciados mediante o desenho de Solomon. A carpoteca quando usada na escola promoveu aos alunos um maior número de acertos nos testes propostos se mostrando eficiente como material didático, pois proporcionou a manipulação das estruturas e assimilação do conteúdo devendo ser utilizada de forma complementar às aulas teóricas.
\end{abstract}

PALAVRAS-CHAVE: Coleção de frutos. Ensino de botânica. Material didático. 


\section{Introdução}

Tendo em vista a dificuldade encontrada para ministrar aulas de ciências e biologia na educação básica, os professores recorrem frequentemente a recursos didáticos como meios de aprendizagem e fixação dos conteúdos. Essas ferramentas despertam a curiosidade pela temática, além de trabalhar outros valores importantes na formação psicológica dos alunos (MENDES et al, 2011). Nesse sentido, fazendo uso de recursos didático-pedagógicos, os educadores buscam compensar as falhas acarretadas pelo ensino tradicional, pois promovem a exposição do conteúdo de forma diferenciada, além de introduzirem de forma participativa os alunos no processo de aprendizagem (CASTOLDI e POLINARSKI, 2009).

O ensino convencional de botânica apresenta uma aula teórica, onde o aluno geralmente memoriza um conjunto de informações como sendo os conteúdos mais importantes, ainda que estes possam não estar inseridos em sua realidade (RISSI e CAVASSAN, 2013), ademais, no ensino de ciências e biologia, a maioria dos professores limita-se ao uso do livro didático, caracterizando-o não apenas como um recurso auxiliar (MARANDINO et al, 2009). Logo, o que é ensinado e a forma como se ensina, influenciam os alunos a perceberem a biologia como uma disciplina relevante e merecedora de atenção, ou como uma disciplina pouco atrativa (KRASILCHIK, 2004).

Além disso, a capacidade de transmissão do conhecimento do professor e do conteúdo dos livros constituem uma condição necessária, mas não suficiente para garantir a aprendizagem, visto essa representar um processo de assimilação e construção de conhecimentos e habilidades (TAROUCO et al, 2004). Nesse contexto, o livro didático não atribui muita ênfase ao estudo de frutos, quando comparado a outros órgãos vegetais como raízes, caules e folhas (AMADEU; MACIEL, 2014), estando também a conquista do conhecimento em botânica afetada pela ausência de interação estabelecida com as plantas, assim como a falta, 
ou ainda a má utilização de equipamentos, métodos e tecnologias que podem contribuir para o aprendizado (ARRUDA e LABURÚ 1996; CECCANTINI, 2006).

Para Resende et al. (2002) o aprendizado se mostra mais efetivo quando o aluno tem à sua disposição o material objeto de estudo. Assim, as coleções biológicas desenvolvem no âmbito do ensino formal de biologia, uma função primordial, que corresponde a sua utilização como material didático (AZEVEDO et al, 2012). Em biologia, as coleções de herbário podem ser apontadas como uma valiosa estratégia para desenvolver conceitos, pois permitem a manipulação das plantas e suas estruturas, de forma a tornar a aprendizagem mais envolvente e instigante (FAGUNDES, 2006). Desta forma a carpoteca (do grego carpo = fruto, teça = loja) resguarda uma coleção de frutos usualmente auxiliar dos herbários, e tem por função básica subsidiar os estudos taxonômicos, ecológicos, fisiológicos, de botânica econômica, etnobotânica e outras áreas do conhecimento científico (RODRIGUES et al, 2002).

Ao longo da história, coleções biológicas têm sido depósitos estáticos de informação, catalogando espécimes e realizando atividades de análise sistemática. Com demanda por dados sobre espécies e espécimes de diferentes disciplinas e áreas de conhecimento, todavia, as coleções não devem ser vistas unicamente à constatação da existência de determinados organismos no passado. Sua missão deve ser a de documentar, compreender e educar o mundo sobre a vida em nosso planeta, no passado e no presente (CANHOS et al, 2006). Rissi e Cavassan (2013) mostram essa nova tendência no ensino em desenvolver trabalhos que visam a utilização de atividades pedagógicas diferenciadas como estratégias inovadoras para o ensino de biologia. Exemplos de atividades pedagógicas são os modelos didáticos propostos por Ferreira et al. (2013) com modelo de junção intercelular desmossomo no ensino de biologia celular, Matos et al. (2009) com modelos didáticos entomológicos e Portugal et al. (2011) apresentou modelo sintético de pelve no ensino de anatomia. Sendo assim, objetivou-se neste estudo, produzir uma carpoteca e proporcionar a alunos do ensino médio o contato prático com os diferentes tipos de frutos, com o intuito de investigar a eficiência dessa coleção botânica no processo de ensino aprendizagem acerca do estudo dos frutos.

\section{Metodologia}

\section{Produção da carpoteca}

As coletas dos frutos e a aplicação da carpoteca no âmbito escolar foram realizadas no município de Picos-PI, no Semiárido brasileiro. A referente cidade está 
localizada na região centro-sul do Piauí e é cortada pelo rio Guaribas, estando sua sede geograficamente marcada nas coordenadas geográficas $07^{\circ} 04^{\prime} 37^{\prime \prime}$ latitude sul e 41 28' 01" longitude oeste (AGUIAR e GOMES, 2004; ROCHA et al, 2015). Com clima tropical quente, é característico do município uma dupla estacionalidade climática bem definida, ocorrendo um período chuvoso de dezembro a março e outra estação seca de abril a novembro (OLIVEIRA et al, 2014). A vegetação de caatinga é predominante na região, entretanto, extensas áreas de cerrado e babaçuais também estão presentes (FERNANDES e BEZERRA, 1990).

Nas coletas foi adotado o método de caminhada pelas estradas da região, com foco na procura por frutos maduros, sendo anexado para cada espécime o local da coleta, o hábito da planta e informações básicas com relação a morfologia, para facilitar a identificação da espécie e caracterização do fruto. O material coletado foi encaminhado ao Campus Senador Helvídio Nunes de Barros, da UFPI, no Laboratório de Limnologia e Botânica onde houve o processamento dos frutos. Os frutos carnosos foram preservados em álcool $70 \%$ em recipientes de vidro fechados hermeticamente e os frutos secos foram expostos ao sol, e uma vez desidratados, também foram armazenados em potes de vidro. A classificação dos frutos baseou-se em W. Vidal e M. Vidal (2000) e informações como nome popular, espécie e família, tipo do fruto, número de sementes, consistência do pericarpo e informação quanto à deiscência dos frutos foram registradas em etiquetas colocadas em cada recipiente.

\section{Carpoteca como ferramenta didática}

A carpoteca foi testada como ferramenta didática na Unidade Escolar Landri Sales. Para isso a Diretora da escola localizada na Rua Monsenhor Hipólito no 959 no Bairro Centro, Picos - PI, foi inicialmente esclarecida sobre os objetivos desta pesquisa e autorizou a aplicação desta atividade nas turmas de segundo ano do ensino médio. No processo de coleta de dados foram levadas em consideração as exigências contidas na Resolução 196/96 do Conselho Nacional de Saúde, que regulamenta a pesquisa com seres humanos (BRASIL, 1996). Para tanto, os participantes foram anteriormente esclarecidos, sobre os objetivos desta pesquisa, ficando livres para colaborar com a mesma. Esse estudo não possui nenhum conflito de interesse.

Selecionou-se aleatoriamente 60 alunos os quais foram divididos Solomon (1949) e denominados G1, G2, G3 e G4. No intuito de possibilitar aos 
participantes um esclarecimento sobre o conteúdo, foi ministrada uma aula sobre os frutos e as principais classificações destes órgãos vegetais usando o auxílio de uma apresentação em PowerPoint como recurso visual. Como instrumento de avaliação foram utilizados os seguintes questionários: Pré-teste, para verificação do conhecimento prévio dos alunos e o pós-teste para a verificação do conhecimento adquirido após as aulas e à carpoteca. O conteúdo dos testes foi um questionário com uma sequência de 10 questões de múltipla escolha, com apenas uma alternativa considerada correta. As perguntas estavam relacionadas à origem e constituição dos frutos, suas variações morfológicas e consequentemente à classificação tipológica dos mesmos.

Desta forma, os quatro grupos sofreram tratamentos distintos (figura 1). 0 G1 respondeu ao pré-teste, assistiu a aula e respondeu ao pós-teste sem entrar em contato com a carpoteca. Já o G2 não respondeu ao pré-teste, assistiu a aula e respondeu o pós-teste, sem também entrar em contato com a carpoteca. O G3 respondeu ao pré-teste, assistiu aula, observou a carpoteca e respondeu ao pósteste e o G4 assistiu a aula, observou a carpoteca e respondeu somente o pós-teste. Para a comparação dos resultados foram atribuídas médias gerais do número de acertos dos participantes. A apresentação da carpoteca nos grupos G3 e G4 seguiu a classificação destes mediante a origem, onde foram separados os frutos simples, pseudofrutos, múltiplos e compostos. Logo depois, foram visualizados os tipos encontrados em cada grupo, estabelecendo a relação entre o nome característico do tipo do fruto, suas características morfológicas e o nome vulgar do mesmo. Paralelamente à observação tipológica dos frutos, seguiu-se a retomada de outras informações trabalhadas na aula teórica, como o número de sementes, o mecanismo de abertura e consistência do pericarpo para cada fruto apontado.

Figura 1. Representação esquemática dos tratamentos aplicados nos 4 grupos.

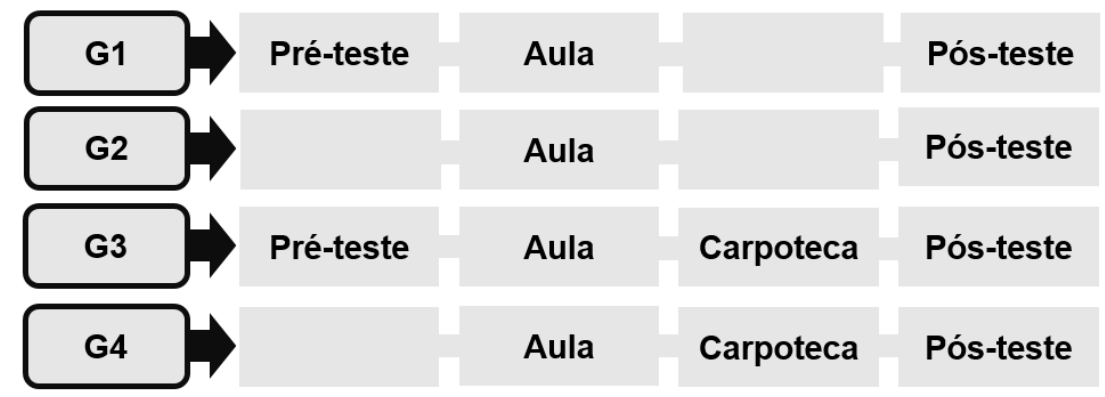

(Fonte: autor da pesquisa) 


\section{Resultados e discussão}

As médias aritméticas foram calculadas e dispostas na Tabela 1, de tal modo, percebe-se em G1, que a média obtida no pré-teste foi 4,5 , sendo notificado seguidamente no pós-teste um aumento no número de questões corretas, com a média de acertos igual a 5,5. Para G2, onde não foram aplicados pré-testes, a média atingida no pós-teste deteve o valor de 4,6. No grupo G3 o teste inicial apontou 3,9 de média, diferente do encontrado no pós-teste, com 7,1. Já para G4, onde apenas o teste final foi aplicado aos participantes, a nota recolhida foi 7,0 .

Tabela 1. Médias obtidas em cada grupo no pré-teste e no pós-teste.

\begin{tabular}{|c|c|c|}
\cline { 2 - 3 } \multicolumn{1}{c|}{} & \multicolumn{2}{c|}{ Notas } \\
\hline Grupos & Pré-teste & Pós-teste \\
\hline G1 & 4,5 & 5,5 \\
\hline G2 & Não aplicado & 4,6 \\
\hline G3 & 3,9 & 7,1 \\
\hline G4 & Não aplicado & 7,0 \\
\hline \multicolumn{2}{|c|}{ (Fonte: autor da pesquisa) } \\
\hline
\end{tabular}

Em todos os grupos foi apresentada a aula sobre o tema com o auxílio de slides, visto ser os recursos audiovisuais usados no ensino de ciências eficientes, pois exigem dos alunos coordenações simbólicas de decodificação necessárias à real percepção do conhecimento (ROSA, 2008). Assim, no grupo G1 que passou pela aula, mas não teve a carpoteca à disposição, observou-se que algumas informações puderam ser agregadas frente ao conhecimento prévio dos alunos. Entretanto, ao comparar G1 e G2, foi evidenciada uma influência do pré-teste na resolução do pósteste no primeiro grupo, pois a média do teste final em G2, que também passou pela aula, foi aproximada da nota atingida no teste inicial de G1.

Em G1 e G3, submetidos ao pré-teste foram notificadas nesses as menores médias, declarando assim uma carência no conhecimento dos discentes acerca do conteúdo de morfologia e tipologia de frutos. Nesse sentido, frente aos conhecimentos específicos que contemplam os conteúdos repassados em sala de aula, percebe-se existir uma deficiência de informações voltadas ao estudo do reino Plantae (PAES et al, 2015). Destarte, é evidente a necessidade de aulas mais instigantes, como aquelas que colocam o aluno diretamente em contato com seu 
objeto de estudo, pois assim, é despontado mais qualidade de ensinoaprendizagem levando a uma maior absorção de conhecimento pelos alunos, bem como, para o professor. (ALBINO et al, 2016).

Observando as médias nos grupos submetidos ao tratamento com a carpoteca, foram atingidos os maiores valores no estudo, logo, a carpoteca pode ser reconhecida como um eficiente recurso didático no desenvolvimento de aulas de botânica, com foco no estudo dos frutos, pois, a coleção desses órgãos propiciou uma redução das dificuldades encontradas no tocante à nomenclatura infrequente no cotidiano do alunado. Em conformidade com Paes et al. (2015), entende-se que as atividades práticas geram motivação e aprendizagem por meio da interação com o meio ambiente, o que terminar por suprir dificuldades encontradas pelos alunos quanto ao entendimento de conceitos específicos da botânica.

Foi notável o crescimento do número de questões corretas após a aula ministrada, entretanto com a carpoteca obteve-se um aumento acentuado no número de acertos, uma vez que a referida atividade pode ser vista como inovadora para os alunos, corroborando com Gonçalves e Morais (2011), que apontam a utilização de recursos e a manipulação das estruturas vegetais in vivo como responsáveis por tornar a aula mais atrativa. A atividade despertou o interesse dos alunos perante a observação da coleção de frutos e consequentemente garantindo uma menor abstração sobre o conteúdo trabalhado, de modo que tornou-se possível relacionarem o conteúdo teórico com a diversidade vista na carpoteca, logo, para Bock et al. (2009) uma aprendizagem significativa é aquela em que o estudante assimila o conteúdo e relaciona com conceitos relevantes, claros e disponíveis na estrutura cognitiva, onde uma disciplina não pode ser desenvolvida apenas de forma teórica, mas apoiada em aulas práticas que contribuam para aprimorar os conhecimentos.

A carpoteca usada na educação básica pode ser apontada como importante material didático com foco no estudo dos frutos, pois promove uma maior proximidade entre os estudantes e o material de estudo, uma vez que, apenas pelo livro a interação entre o educando e os órgãos vegetais pode se mostrar insuficiente para garantir sucesso no processo de ensino e aprendizagem de botânica. Perticarrari et al. (2011) afirmam que a observação de uma estrutura vegetal articulada com a teoria mostra-se eficiente ferramenta de aprendizagem pois atividades investigativas, que aproximem os jovens do seu objeto de estudo podem ajudá-los a aprender conceitos vistos em botânica, colocando-os na situação de 
construtores do conhecimento, sendo importante a experimentação que relaciona teoria e prática.

A presença dos frutos foi importante para a observação e comparação da variação morfológica que é determinante para classificação tipológica, onde os termos empregados foram melhor relacionados, apoiando dessa forma a recomendação de Joly (1976), que o professor ao trabalhar a classificação das plantas recorra à utilização de exemplares vivos. Para Matos et al. (2009) a utilização de metodologias alternativas para o ensino deve ser estimulada nas instituições de ensino do país, no sentido de se promover a integração entre os conteúdos abordados nas disciplinas com o desenvolvimento de atividades práticas, possibilitando assim a intensa participação dos alunos no processo de aprendizagem.

Na literatura não foram encontradas comparações entre o uso da carpoteca e sua aplicação no ensino médio voltado ao estudo morfológico e tipológico dos frutos. Ceccantini (2006) afirmou que modelos didáticos permitem a compreensão tridimensional das estruturas e podem auxiliar a sanar deficiências nos recursos didáticos. Machado-Júnior e Acrani (2010) relataram os efeitos de um minicurso de botânica para alunos do ensino médio, e segundo os autores, a apresentação do material in vivo que foi usado no reconhecimento e classificação dos vegetais se mostrou um importante material didático, pois a adoção de atividades que acompanhem procedimentos práticos e relacionem à realidade dos alunos, denotam significado e importância ao assunto trabalhado, estimulam a curiosidade e despertam o senso crítico dos alunos. De modo semelhante, Lopes et al. (2011) realizaram uma oficina envolvendo análise das características de plantas medicinais e concluíram que a atividade contribuiu para aguçar o interesse dos alunos, já que os discentes puderam interagir e questionar ao manusearem as plantas.

Desse modo, a aplicação da carpoteca no âmbito do ensino médio proporcionou a manipulação das estruturas, a assimilação do conteúdo e a construção de conhecimentos, devendo ser utilizada de forma complementar às aulas teóricas tornando-se um material significativo para o ensino da botânica, principalmente no estudo dos frutos. Assim, a carpoteca pode contribuir para a elucidação de aulas práticas, facilitando a compreensão dos assuntos por parte dos alunos (SCHAFFER e LÍBANO, 2011).

\section{Conclusões}


Frente ao exposto, podem ser entendidos os benefícios da utilização de atividades diversificadas nas aulas de botânica, ao passo que, relacionado ao conteúdo dos frutos, a introdução de uma carpoteca na sala de aula desperta o interesse dos alunos e também contribui para a fixação e aplicação prática dos conteúdos trabalhados nas aulas teóricas, além do mais, a carpoteca constituída por frutos de uma determinada região facilita a familiarização do assunto dos livros com o meio ambiente no qual os alunos estão inseridos.

\section{Agradecimentos}

À direção da Unidade Escolar Landri Sales e aos professores de biologia da instituição pelo acolhimento e auxilio prestado, bem como aos alunos que participaram do trabalho. 


\title{
Fruit collection how teaching resource in botany
}

\begin{abstract}
The teaching of botany is done with a traditional approach that cause disinterest in students, and is still marked by other problems such as lack or misuse of teaching resources or little interaction between students and plants. The objective of this work was to produce one fruit collection investigate the effectiveness of this botanical collection in the teaching process learning about the fruits study. Through walk method, fruits were collected in Picos city, and in high school classes, 60 students were divided into four groups and have undergone different treatments by the Solomon test. The collection when used in school promoted to students a greater number of correct answers in the proposed tests, being effective as teaching material, because it provided the manipulation of structures and assimilation of content, so it should be used to complement the theoretical classes.
\end{abstract}

KEYWORDS: Biological collection. Didactic resources. Teaching of botany. 


\section{Referências}

AGUIAR, R. B.; GOMES. J. R. C. Projeto cadastro de fontes de abastecimento por água subterrânea, estado do Piauí: diagnóstico do município de Picos. Fortaleza: CPRM - Serviço Geológico do Brasil. 2004. 32 p.

ALBINO, A. M.; FIALHO, S. N.; CHAGAS, M. C.; MARTINS, A. J.; LIMA, R. A. O transmitir da botânica de uma forma multidisciplinar em uma escola pública de Porto Velho-RO. South American Journal of Basic Education, Technical and Technological, v. 3, n. 1, p. 10-17, 2016.

AMADEU, S. O.; MACIEL, M. D. A importância da transposição didática no ensino da morfologia vegetal no estudo dos frutos. Revista de Produção Discente em Educação Matemática, v. 3, p. 82-90, 2014.

ARRUDA, S. M.; LABARU, C. E. Considerações sobre a função do experimento no ensino de ciências. Ciência \& Educação, v. 3, p. 14-24, 1996.

AZEVEDO, H.; FIGUEIRÓ, R.; ALVES, D. R.; VIEIRA, V.; SENNA, A. R. O uso de coleções zoológicas como ferramenta didática no ensino superior: um relato de caso. Revista Práxis, v. 4, p. 43-48, 2012.

BOCK, A. M.; FURTADO, O.; TEIXEIRA, M. L. T. Psicologias: uma introdução ao estudo da psicologia. São Paulo: Saraiva, 2009. 365p.

CANHOS, D. A. L. et al. Coleções biológicas e sistemas de informação. In: MINISTÉRIO DA CIÊNCIA E TECNOLOGIA. Diretrizes e estratégias para a modernização de coleções biológicas brasileiras e a consolidação de sistemas integrados de informação sobre biodiversidade. Brasília: CGEE/MCT, 2006. p. 241 314.

CASTOLDI, R; POLINARSKI, C. A. A utilização de Recursos didático-pedagógicos na motivação da aprendizagem. In: II, Ponta Grossa, PR, 2006, Anais... Simpósio Nacional de Ensino de Ciência e Tecnologia, 2006.

CECCANTINI, G. Os tecidos vegetais têm três dimensões. Revista Brasileira de Botânica, São Paulo,v. 29, n. 2, p. 335-337, 2006.

FAGUNDES, J. A. Herbário escolar: suas contribuições ao estudo da Botânica no 
FERNANDES, A.; BEZERRA, P. Estudo fitogeográfico do Brasil. Stylos Comunicações, Fortaleza, 1990.

FERREIRA, P. M. P.; MOURA, M. R.; COSTA, N. D. J.; SILVA, J. N.; PERON, A. P.; ABREU, M. C. de; PACHECO, A. C. L. Avaliação da importância de modelos no ensino de biologia através da. Revista Brasileira de Biociências, v. 11, n. 4, p. 388-394, 2013. GONCALVES, H. F.; MORAES, M. G. Atlas de anatomia vegetal como recurso didático para dinamizar o ensino de Botânica. Enciclopédia Biosfera, v. 7, p. 1608-1618, 2011.

JOLY, A. B. Botânica: introdução à taxonomia vegetal. Nacional, São Paulo, 1976. KRASILCHIK, M. Prática de Ensino de Biologia. Editora da Universidade de São Paulo, 2004.

LOPES, I. S.; GUIDO, L. de F. E.; CUNHA, A. M. O.; JACOBUCCI, D. F. C. Estudos coletivos de educação ambiental como instrumento reflexivo na formação continuada de professores de ciências em espaços educativos formais e nãoformais. Revista Electrónica de Enseñanza de las Ciencias, v. 10, p. 516-530, 2011.

MACHADO JUNIOR, A.; ACRANI, S.; CARNEIRO, N. M. Atividades práticas de botânica comomeio de aproximação entre as instituições de ensino superior, a formação docente e o ensinomédio estadual. Revistada SBEnBIO, v. 3, p. 22352242, 2010.

MARANDINO, M.; SELLES, S. E.; FERREIRA, M. S. Ensino de biologia: histórias e práticas em diferentes espaços educativos. São Paulo: Cortez, 2009.

MATOS, C. H. C.; OLIVEIRA, C. R. F. SANTOS, M. P. F.; FERRAZ, C. S. Utilização de Modelos Didáticos no Ensino de Entomologia. Revista de Biologia e Ciências da Terra, v. 9, n. 1, p. 19-23, 2009.

MENDES, M. B. P. ; FIGUEIREDO, A. Q. S. A. ; BRANDAO, R. A. ; CARDOSO, A. L. B. D. . Integrando palavras: uma nova abordagem didática para o ensino de Botânica na escola. Brasília: Pontes Editores, 2011, Anais... XIV Congresso Internacional de Humanidades, 2011.

OLIVEIRA, Y. R.; SILVA, P. H.; ABREU, M. C. Análise tipológica dos frutos do município de Picos, Piauí, Brasil. In: XI Semana Nacional de Ciência e Tecnologia IV Feira Estadual de Ciência e Tecnologia, 2014, Picos. Anais... XI Semana Nacional de 
PAES, L. S.; LIMA, D. C. F.; MARQUES, J. D. O.; AZEVEDO, R. O. M.; BARBOSA, T. J. V. B. Atividades didáticas para o ensino da classificação das plantas no sétimo ano do ensino fundamental. Investigação Qualitativa em Educação, v. 2, 2015.

PERTICARRARI, A.; TRIGO, F. R.; BARBIERI, M. R. A contribuição de atividades em espaçosnão formais para a aprendizagem de botânica de alunos do Ensino Básico. Ciência em Tela, v.4, p. 1-12, 2011.

PORTUGAL, H. S. P.; PALMA, P. C. R.; FRAGA, R. de; RICCETTO, C. L. Z.; ROCHA, S.; CARIAS, L. Modelo pélvico sintético como uma ferramenta didática efetiva comparada à pelve cadavérica. Revista Brasileira de Educação Médica, v. 35, n. 04, p. 502-506, 2011.

RESENDE, A. L.; FERREIRA, J. R.; KLOSS, D. F. M.; NOGUEIRA, J. D.; ASSIS, J. B. de. Coleções de animais silvestres, fauna do cerrado do sudoeste goiano, o impacto em educação ambiental. Apadec, v. 6, n. 1, p.35-41, 2002.

RISSI, M. N.; CAVASSAN, O. Uma proposta de material didático baseado nas espécies de Vochysiaceae existentes em uma trilha no cerrado de Bauru - SP. Biota Neotropica, v. 13, n. 1, p. 27-41, 2013.

ROCHA, L. A.; ROCHA, A. M.; PACHECO, A. C. L.; ABREU, M. C. Diferenças foliares morfoanatômicas de quatro espécies da família Anacardiaceae. Caderno de Pesquisa. Série Biologia (UNISC), v. 27, n. 2, p. 35-48, 2015.

RODRIGUES, S. T.; Potiguara, R. C. de V.; FERREIRA, G. C.; SILVA, J. Y T S. Acervo do Herbário lan-Carpoteca. Belém: Embrapa Amazônia Oriental, 2002.

ROSA, P. R. S. Instrumentação para o Ensino de Ciências. Departamento de física - UFMS, Campo grande, 2008.

SCHAFFER, C. C.; LIBANO, A. M. Tipologia de frutos e síndromes de dispersão de um fragmento de Cerrado sensu stricto da APA do Gama e Cabeça de Veado e montagem de coleção didática de frutos. Universitas-Ciências da Saúde, v. 09, p.29-46, 2011.

SOLOMON, R. L. An extension of control group design. Psychological Bulletin, v. 46, 137-150, 1949.

TAROUCO, L. M. R; ROLAND, L. C; FABRE, M-C. J. M; KONRATH, M. L. P. Jogos Educacionais. CINTED, v. 2, n. 1, 2004.

VIDAL, W. N.; VIDAL, M. R. R. Botânica organografia: quadros sinóticos ilustrados 


\section{RBECT}

Recebido: 2016-08-06

Aprovado: 2017-05-10

DOI: $10.3895 /$ rbect.v10n2.4503

Como citar: OLIVEIRA, Y. R.; SILVA, P. H. DA; DEUS, M. S. M. DE; GONCALVES, N. M. N.; ABREU, M. C.

Carpoteca: ferramenta de ensino em botânica. Revista Brasileira de Ensino de Ciência e Tecnologia, v. 10, n.

2, 2017. Disponível em: <https://revistas.utfpr.edu.br/rbect/article/view/4503>. Acesso em: xxx.

Correspondência:

Direito autoral: Este artigo está licenciado sob os termos da Licença Creative Commons-Atribuição 4.0 Internacional. 